Math. Model. Nat. Phenom.

Vol. 2, No. 2, 2007, pp. 56-76

\title{
Recent mathematical results on combustion in hydraulically resistant porous media
}

\author{
P. Gordon ${ }^{1}$ \\ Department of Mathematical Sciences, New Jersey Institute of Technology, \\ University Heights, Newark, NJ 07102
}

\begin{abstract}
Gaseous detonation is a phenomenon with very complicated dynamics which has been studied extensively by physicists, mathematicians and engineers for many years. Despite many efforts the problem is far from a complete resolution. Recently the theory of subsonic detonation that occurs in highly resistant porous media was proposed in [4]. This theory provides a model which is realistic, rich and suitable for a mathematical study. In particular, the model is capable of describing the transition from a slowly propagating deflagration wave to the fast detonation wave. This phenomena is known as a deflagration to detonation transition and is one of the most challenging issues in combustion theory. In this paper we will present some recent mathematical results concerning initiation of reaction in porous media, existence and uniqueness of traveling fronts, quenching and propagation.
\end{abstract}

Key words: subsonic detonation, deflagration to detonation transition, traveling fronts, quenching

AMS subject classification: $35 \mathrm{~K} 57,80 \mathrm{~A} 25$

\section{Introduction}

Premixed gas combustion is the combustion of gaseous reactants which are perfectly mixed prior to ignition. The most distinctive feature of premixed combustion is its ability to assume the form of a self-sustained reaction front propagating subsonically or supersonically at a well-defined speed. Apart from their technological relevance, combustion waves constitute a truly fascinating dynamical system, displaying an amazingly rich variety of phenomena

\footnotetext{
${ }^{1}$ Corresponding author. Email: peterg@njit.edu
} 
such as non-uniqueness of possible propagation regimes, their birth and destruction, chaotic self-motion and fractal-like growth [2], [10],[38],[42].

As is well known, there are basically two mechanisms controlling propagation of combustion waves in gaseous mixtures: molecular transport and adiabatic compression. The first mechanism pertains to deflagrative combustion or deflagration and the second to detonation. For realistic materials, the characteristic deflagration velocity is relatively low and lies between a few centimeters and a few meters per second. By contrast, the velocity of detonation exceeds deflagration velocity by several orders of magnitude. Normally, the adiabatic compression is provided by a shock and the resulting combustion wave (detonation) spreads at a supersonic speed. In conventional detonation, the shock and the adiabatic compression are two inseparable ingredients. However, the coupling between the two is not inevitable. In hydrodynamically resistant flows, as those which develop in porous beds, the burning velocity may fall significantly below its thermodynamic, Chapman Jouguet (CJ), value, and under certain conditions, the propagation may well become subsonic and therefore shockless [32], [33], [37], [39]. The shockless propagation still involves pressure peaks and is sustained

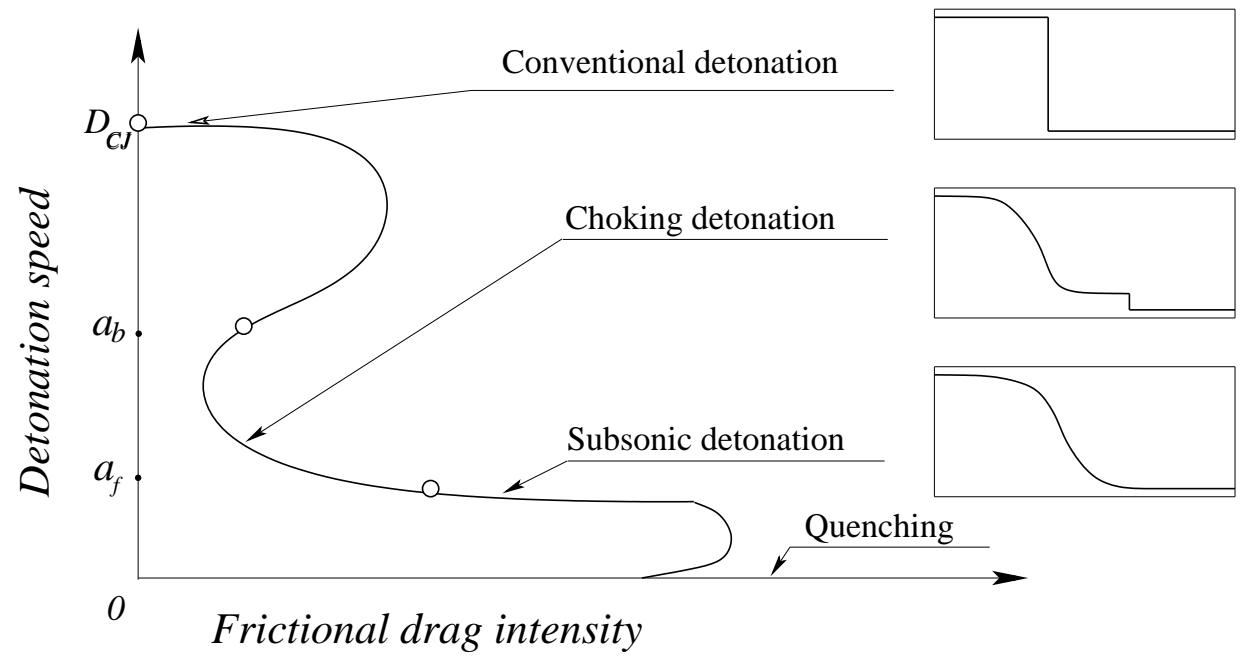

Figure 1: Detonation speed in hydraulically resistant porous media and typical profiles of detonation fronts. Here $D_{C J}, a_{b}$ and $a_{f}$ correspond to Chapman Jouguet detonation and sound speeds in a fresh and burned gas receptively.

by adiabatic compression which is now spread by the drag-induced diffusion of pressure. The transition from supersonic to subsonic propagation normally occurs in a gradual manner, e.g., by dilution of the mixture. In the near-sonic propagation regime, the presence or absence of shocks is not crucial and there is no major distinction between supersonic and subsonic combustion [25]. The subsonic mode emerges as a continuous extension of the supersonic one, and hence, may well be referred to as subsonic detonation, two words that one would have thought could never be connected! The idea of extending the concept of detonation over the subsonic domain is not new and has already been discussed by Mitrofanov in his 1987 review on non-classical combustion waves [37]. It is interesting that the issue was raised by 
the experimentalists ten years before the theoretical substantiation of the phenomenon. In [6] and [7], employing the classical Zeldovich-Fanno [43] formulation, it was shown that the conventional and subsonic modes are actually two special cases of the general drag-affected detonation, and in the velocity-drag plane belong to the same curve of dynamically feasible states, see Fig. 1. The conventional CJ-detonation corresponds to the limit of low hydraulic resistance, whereas the subsonic detonation corresponds to the limit of strong resistance.

In this paper we will present some recent mathematical results concerning subsonic detonation. The paper is organized as follows. In section 2, we briefly describe the simplest model for studying subsonic detonation and major physical effects that this model can describe. In section 3, reductions of the model will be discussed. Section 4 is devoted to existence, uniqueness and qualitative properties of traveling wave solutions. In section 5, we present results on quenching, lock of propagation as well as bounds on propagation velocity. Finally, in section 6 , we present the model of stabilization of reaction in a presence of cold boundary which is a natural generalization of Gelfand's problem for porous media.

\section{Subsonic detonation. Mathematical model and major physical effects}

Perhaps the physically simplest and yet experimentally feasible system for studying subsonic detonation is combustion in inert porous medium [32]. In this case, on the one hand, the distortions introduced by the porous matrix may be ignored while, on the other hand, the resistance of the matrix to the gas flow is often so strong that one may neglect the inertial effects and take Darcy's law as the momentum equation. In this high drag limit, the shocks are ruled out and the pressure nonuniformities are equalized not by the acoustic waves but rather through the diffusion of pressure associated with low Reynolds number creeping flows.

To single out the impact of momentum loss, the effective features of the reactive gasporous medium system can be assumed to be controlled exclusively by its gaseous phase subjected to the resistance of the porous medium matrix. As an additional simplification, the so-called small-heat-release approximation [36] may be employed where variations of temperature, pressure, density and gas velocity are regarded as small and, hence, the nonlinear effects are ignored everywhere but in the reaction rate term, which is generally highly sensitive even to minor temperature changes. Under these assumptions the resulting model reads [4],

$$
\begin{aligned}
& T_{t}-\left(1-\gamma^{-1}\right) P_{t}=\varepsilon \Delta T+Y F(T), \\
& P_{t}-T_{t}=\Delta P, \\
& Y_{t}=\varepsilon L e^{-1} \Delta Y-\gamma Y F(T) .
\end{aligned}
$$

Here T, $P$ and $Y$ are the appropriately normalized temperature, pressure and concentration of the deficient reactant, $\gamma>1$ is the specific heat ratio, $\varepsilon$ is the ratio of thermal and pressure diffusivities, $L e$ is a Lewis number and $Y F(T)$ is the normalized reaction rate. 
The first and the last equations in (2.1) represent the partially linearized conservation equations for energy and for deficient reactant, while the second follows from the linearized continuity equation, and equations of state and momentum.

The problem $(2.1)$ is posed in the domain $\Omega$. The simplest physically relevant domains where the problem can be considered are either a cylinder, that is, $\Omega=\mathbb{R} \times D$, where $D$ is bounded domain in $\mathbb{R}^{d-1}$ with $d=1,2,3$ with Neumann boundary conditions of $\partial D$ or in entire space $\Omega=\mathbb{R}^{d}$.

Throughout this paper we will assume that nonlinearity $F(T)$ is of the combustion type with ignition temperature [42],[43]. Specifically,

$$
F(T)=0 \text { for } \quad 0 \leq T<T^{*}
$$

Moreover, $F(T)$ is an increasing Lipschitz continuous function for $T \geq T^{*}$, except for a possible discontinuity at the ignition temperature $T=T^{*}$. This type of nonlinearity is one

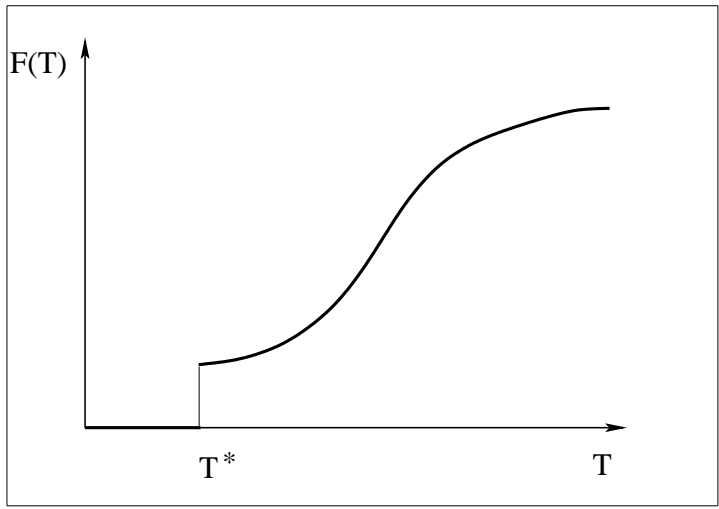

Figure 2: Combustion type nonlinearity with the ignition temperature.

of the most common in the combustion theory [40],[43]. A typical example of $F(T)$ is the Arrhenius law [41]

$$
F(T)=\left\{\begin{array}{cll}
\exp \left(\frac{Z\left(T-\left(1-\gamma^{-1}\right)\right)}{\sigma+(1-\sigma) T}\right) & \text { if } & T \geq T^{*} \\
0 & \text { if } & T<T^{*}
\end{array}\right.
$$

where $Z$ is a scaled activation energy (Zeldovich number) and $0<\sigma<1$ is a ratio of characteristic temperatures of the fresh and burned gases.

One of the central problem in the combustion theory is the initiation of combustion process. In many cases, initiation occurs by localized energy deposition (hot spot). In the framework of the model (2.1), this corresponds to the following initial conditions [22]

$$
T(0, \mathbf{x})=T_{0}(\mathbf{x}) \geq 0, \quad Y(0, \mathbf{x})=1, \quad P(0, \mathbf{x})=0
$$

That is, the entire domain $\Omega$ is initially filled uniformly with the reactant, pressure field prior to ignition is also uniform, while temperature is spatially localized. 
It is important to note that for many gas-porous medium systems the gas pressure diffusivity exceeds its thermal diffusivity by several orders of magnitude, thereby making $\varepsilon$ a natural small parameter of the problem. For real world porous media $\varepsilon$ varies in the range $\varepsilon \sim 10^{-2}-10^{-5}[38]$. As will be shown below, the smallness of $\varepsilon$ gives rise to very interesting dynamics in the system (2.1).

In order to investigate possible scenarios of propagation, it is convenient to introduce the bulk burning rate. Following [12], we define a bulk burning rate as an appropriately scaled average value of the time derivative of the temperature, which in case of the system (2.1), coincides with the average of the nonlinear term $Y F(T)$ evaluated on the solution of the problem (2.1) over domain $\Omega$. When $\Omega$ is a cylinder, the bulk burning rate assumes a convenient form

$$
V(t)=\frac{1}{\operatorname{Area}(D)} \int_{\Omega} T_{t}(\cdot, t)=\frac{1}{\operatorname{Area}(D)} \int_{\Omega} Y(\cdot, t) F(T(\cdot, t)),
$$

where $\operatorname{Area}(D)$ is the area of the cross section of the cylinder. This quantity coincides with the propagation velocity of the front, when evaluated on a traveling wave solution. However, it is defined for very general initial data and regimes of propagation.

The dynamics of the system (2.1) with initial conditions (2.4) strongly depends on the support of initial data for temperature. According to numerical simulations [5],[38], the system exhibits an interesting behavior even in the one dimensional case. Starting with a one parameter family of compactly supported initial data $T_{0}(x)=H_{[-L, L]}(x)$, where $H_{[-L, L]}(x)$ is the characteristic function of the interval $[-L, L]$ or, with initial data strongly decaying at infinity (for example, $T_{0}(x)=\exp \left(-(x / L)^{2}\right)$ ) one numerically observes three general patterns. If $L$ is sufficiently small, the bulk burning rate $V(t)$ goes to zero for $t$ large enough

a)

Initial Temperature Profile

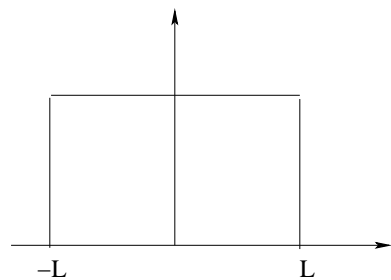

c)

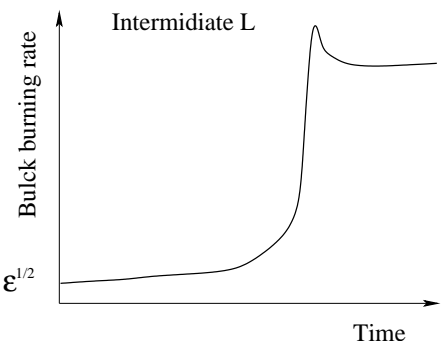

b)

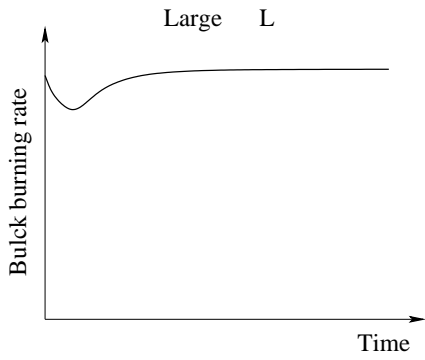

d)

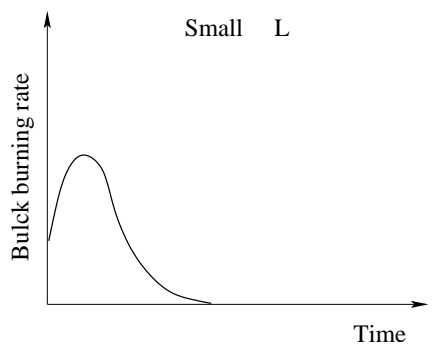

Figure 3: Typical scenarios of propagation dynamics of the system (2.1) with $\varepsilon$ small (see Fig. 3 d). This regime can be called initiation failure or quenching. The second regime 
corresponds to the case of large $L$. In this case, after a very short induction time, the bulk burning rate $V(t)$ becomes essentially constant, and of order unity (see Fig. $3 \mathrm{~b}$ ). The third, and most interesting, case emerges between the two previous regimes when the support $L$ is large enough to prevent quenching but small enough to realize immediate convergence to the traveling front. In this case, the detonation front forms after a long induction time as a result of an abrupt transition from slowly spreading deflagration wave driven by thermal diffusivity. Namely, the bulk burning rate stays almost constant and scales as $\sqrt{\varepsilon}$ for a time interval of the order $1 / \sqrt{\varepsilon}$. Then the solution experiences rapid changes and jumps to the detonation regime where the bulk burning rate assumes the value of order unity, Fig. 3 c. This regime can be interpreted as a transition from deflagration to detonation, the phenomena which still remains one of the most challenging issues in the combustion theory [38], [41], [43]. Indeed, the scaling of the bulk burning rate suggests that the initial period of slow propagation occurs almost exclusively due to thermal diffusivity and, therefore, can be called deflagration. During this part of the evolution both temperature and pressure fields rise and occupy a considerable spatial domain. In other words, the deflagration regime "prepares" the initial data for a successful initiation of transition to the fast regime. After the transition has occurred, the major transport mechanism becomes the pressure diffusivity as can be clearly seen from the scaling of the bulk burning rate. Thus, in this regime, the reaction front is driven mostly by the pressure diffusivity, and therefore, can be called subsonic detonation.

It is really amazing that such a greatly simplified model can still reproduce an effect of transition from deflagration to detonation. In his review on combustion modeling, Sivashinsky wrote [38] : "The frictional mechanism of the [deflagration to detonation] transition was uncovered quite unexpectedly in the course of studies of deflagrative combustion within an inert porous bed under condition of strong hydraulic resistance where the emerging detonation falls into the subsonic range."

We have to note that the three regimes described above may become more complex due to instabilities of the subsonic detonation regime, which under certain conditions, may develop. This was observed in a number of numerical experiments [6],[7]. There is also a possibility of instabilities in the deflagration regime which may lead to a faster transition from deflagration to detonation. However, on a qualitative level, quenching, the immediate formation of detonation wave, and the transition from deflagration to detonation are three basic regimes that were observed in a numerical modeling of the system (2.1) and some of its generalizations [38].

\section{Reductions of the model}

As suggested by numerical simulation at $\varepsilon$ small, one may single out two distinct modes of propagation, deflagration (propagation with characteristic velocity of order $\sqrt{\varepsilon}$ ) and detonation (propagation with the velocity of order one). It was conjectured in [4] that the small thermal diffusivity has no significant influence on the propagation of detonation fronts. In 
other words, it was conjectured that the model (2.1) with $\varepsilon=0$ would adequately describe detonation regime. Substituting this simplification into system (2.1) one has,

$$
\begin{aligned}
& T_{t}-\left(1-\gamma^{-1}\right) P_{t}=Y F(T), \\
& P_{t}-T_{t}=\Delta P \\
& Y_{t}=-\gamma Y F(T) .
\end{aligned}
$$

The system (3.1) can be reduced to a system of two equations. Indeed, adding the first and the last equations of the system (3.1) one obtains a conservation law

$$
\left(T-\left(1-\gamma^{-1}\right) P+\gamma^{-1} Y\right)_{t}=0 .
$$

Integrating this expression with respect to time and taking into account initial conditions (2.4) one has

$$
T(\mathbf{x}, t)=T_{0}(\mathbf{x})+\left(1-\gamma^{-1}\right) P(\mathbf{x}, t)+\gamma^{-1}(1-Y(\mathbf{x}, t)) .
$$

Next, combining the first and the second equations of the system (3.1) we have

$$
P_{t}=\gamma \Delta P+\gamma Y F(T)
$$

Rescaling spacial coordinates $\left(\Delta \rightarrow \gamma^{-1 / 2} \Delta\right)$ we have the following system

$$
\begin{aligned}
& P_{t}=\Delta P+\gamma Y F(T) \\
& Y_{t}=-\gamma Y F(T) \\
& T(\mathbf{x}, t)=T_{0}(\mathbf{x})+\left(1-\gamma^{-1}\right) P(\mathbf{x}, t)+\gamma^{-1}(1-Y(\mathbf{x}, t))
\end{aligned}
$$

which is equivalent to (3.1). Thus, the model of subsonic detonation in the absence of thermal diffusivity reduces to the system of two degenerate reaction diffusion equations. It is important to note the system (3.5) resembles one arising in a study of conventional gasless combustion. The main difference of the system (3.5) and the system describing gasless combustion is that the nonlinear term $F$ in the model (3.5) is a function of two variables (linear combination of $P$ and $Y$ ) in contrast to thermo-diffusive problems where $F$ is a function of single variable. The similar structure of the problem (3.5) and the model of gasless combustion gives some intuition for studying system (3.5). However, one should be aware of the fact that the difference in the nonlinear terms provides not only technical issue in studying the problem (3.5), but also give rise to new effects. For example, the structure of detonation fronts in the so called high activation energy limit is completely different as will be shown in section 4 .

The system $(2.1)$ with $\varepsilon \neq 0$ can also be rewritten in an equivalent form which makes it much more convenient for mathematical study. Moreover, it can be reduced to the system of two reaction diffusion equations in one special case. 
In order to make this reduction let us introduce new functions $S$ and $R$. These two functions are related to the pressure $P$ and temperature $T$ by the linear transformations

$$
\begin{aligned}
& T(t, \mathbf{x})=h_{\varepsilon} S(t, \mathbf{x})+\left(1-h_{\varepsilon}\right) R(t, \mathbf{x}), \\
& P(t, \mathbf{x})=\frac{1}{1-\bar{\varepsilon}}(S(t, \mathbf{x})-\bar{\varepsilon} R(t, \mathbf{x})) .
\end{aligned}
$$

Here, $\lambda_{\varepsilon}$ is a positive solution of the following equation

$$
\left(1-\lambda_{\varepsilon}\right)\left(\gamma+\varepsilon \lambda_{\varepsilon}\right)=1
$$

$\bar{\varepsilon}=\varepsilon\left(1-\lambda_{\varepsilon}\right)^{2}$ and $h_{\varepsilon}=\frac{\lambda_{\varepsilon}}{1-\bar{\varepsilon}}$.

In the new variables $S, R$ and $Y$ and after rescaling spacial coordinates $\Delta \rightarrow\left(1-\lambda_{\varepsilon}\right) \Delta$, the system (2.1) takes the form [21]

$$
\begin{aligned}
& S_{t}=\Delta S+Y F(T), \\
& R_{t}=\bar{\varepsilon} \Delta R+Y F(T), \\
& Y_{t}=\bar{\varepsilon}\left(\left(1-\lambda_{\varepsilon}\right) L e\right)^{-1} \Delta Y-Y F(T), \\
& T(t, \mathbf{x})=h_{\varepsilon} S(t, \mathbf{x})+\left(1-h_{\varepsilon}\right) R(t, \mathbf{x}) .
\end{aligned}
$$

Initial conditions (2.4) in these new variables read,

$$
R(0, \mathbf{x})=\frac{1}{1-\lambda_{\varepsilon}} T(0, \mathbf{x}), \quad S(0, \mathbf{x})=\frac{\bar{\varepsilon}}{1-\lambda_{\varepsilon}} T(0, \mathbf{x}), \quad Y(0, \mathbf{x})=1 .
$$

It is important to note, that the system (3.8) can be significantly simplified in a special case. When $L e=L e^{*}=\left(1-\lambda_{\varepsilon}\right)^{-1}$, adding the second and the third equations of the system (3.8) one can observe that $Y=1-R$ for all times if this condition is satisfied at time $t=0$. Thus the resulting model reads

$$
\begin{aligned}
& S_{t}=\Delta S+(1-R) F(T), \\
& R_{t}=\bar{\varepsilon} \Delta R+(1-R) F(T), \\
& T(t, \mathbf{x})=h_{\varepsilon} S(t, \mathbf{x})+\left(1-h_{\varepsilon}\right) R(t, \mathbf{x})
\end{aligned}
$$

with the initial conditions

$$
R(0, \mathbf{x})=\frac{1}{1-\lambda_{\varepsilon}} T(0, \mathbf{x}), \quad S(0, \mathbf{x})=\frac{\bar{\varepsilon}}{1-\lambda_{\varepsilon}} T(0, \mathbf{x}) .
$$

System (2.1) in the form (3.8) and, especially, in the form (3.10) resembles classical models of the theory of thermo-diffusive combustion [40],[41]. The major difference of the models (3.10) and (3.8) from those appearing in conventional combustion is that the nonlinear term $F$ is a function of two variables $S$ and $R$, whereas in the thermo-diffusive problems, $F$ is a function of a single variable. 


\section{Traveling fronts}

In this section we will present results on existence, uniqueness and some qualitative properties of traveling wave solutions of the problem (2.1). Traveling wave solutions of the problem (2.1) are special solutions of the form

$$
T(x, t)=T_{\varepsilon}(\xi), \quad P(x, t)=P_{\varepsilon}(\xi), \quad Y(x, t)=Y_{\varepsilon}(\xi), \quad \xi=x-c_{\varepsilon} t
$$

where $c_{\varepsilon}$ is the a priori unknown velocity of the traveling wave. Substituting (4.1) into (2.1) we obtain the following system of ODE's

$$
\begin{aligned}
& -c_{\varepsilon} T_{\varepsilon}^{\prime}+c_{\varepsilon}\left(1-\gamma^{-1}\right) P_{\varepsilon}^{\prime}=\varepsilon T_{\varepsilon}^{\prime \prime}+Y_{\varepsilon} F\left(T_{\varepsilon}\right), \\
& P_{\varepsilon}^{\prime \prime}=c_{\varepsilon}\left(T_{\varepsilon}^{\prime}-P_{\varepsilon}^{\prime}\right), \\
& -c_{\varepsilon} Y_{\varepsilon}^{\prime}=\varepsilon L e^{-1} Y_{\varepsilon}^{\prime \prime}-\gamma Y_{\varepsilon} F\left(T_{\varepsilon}\right) .
\end{aligned}
$$

with imposed front-like boundary conditions

$$
\left(P_{\varepsilon}, T_{\varepsilon}, Y_{\varepsilon}\right)(-\infty)=(1,1,0), \quad\left(P_{\varepsilon}, T_{\varepsilon}, Y_{\varepsilon}\right)(+\infty)=(0,0,1)
$$

Solutions of the system (4.2) are indispensable for understanding long time behavior of the system (2.1). Studying these solutions is a standard first step to understand dynamics of reaction diffusion equations.

As discussed in the introduction, numerical simulations suggest that the influence of small thermal diffusivity has no or very minor effect on the propagation of subsonic detonation fronts. Thus, for studying subsonic detonation waves it is very attractive to ignore the thermal diffusivity and formally set $\varepsilon=0$. Within this simplification the system (4.2) assumes the following simplified form

$$
\begin{aligned}
& -c_{0} T_{0}^{\prime}+c_{0}\left(1-\gamma^{-1}\right) P_{0}^{\prime}=Y_{0} F\left(T_{0}\right), \\
& P_{0}^{\prime \prime}=c_{0}\left(T_{0}^{\prime}-P_{0}^{\prime}\right), \\
& -c_{0} Y_{0}^{\prime}=-\gamma Y_{0} F\left(T_{0}\right)
\end{aligned}
$$

with the boundary conditions

$$
\left(P_{0}, T_{0}, Y_{0}\right)(-\infty)=(1,1,0), \quad\left(P_{0}, T_{0}, Y_{0}\right)(+\infty)=(0,0,1)
$$

Solutions of the system (4.4) are well understood. In particular, the following theorem holds.

Theorem 4.1. [22],[14] Assume that $T^{*}<\lambda_{0}$ with $\lambda_{0}=\left(1-\gamma^{-1}\right)$. There is then a unique value of $c_{0}>0$ for which there exists a solution of the problem (4.4). Moreover, functions $P_{0}, T_{0}$ and $Y_{0}$ are monotone functions in $\xi$.

The proof of the Theorem 4.1 consists of two steps. The first step is to show that the solution of the problem (4.4), if it exists, is monotone. This allows the reduction of the 


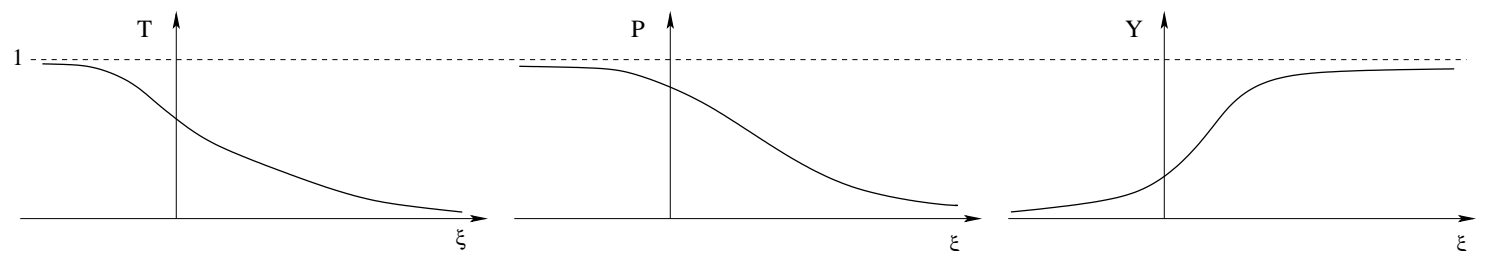

Figure 4: Traveling wave solution for the system (4.4).

problem $(4.4),(4.5)$ to the following first order equation on the phase plane $\left(Y_{0}, P_{0}\right)$

$$
\begin{aligned}
& \frac{d Y_{0}}{d P_{0}}=\frac{\gamma Y_{0} F\left(T_{0}\right)}{c_{0}^{2}\left(1-P_{0}-Y_{0}\right)}, \\
& T_{0}=\left(1-\gamma^{-1}\right) P_{0}+\gamma^{-1}\left(1-Y_{0}\right)
\end{aligned}
$$

with the boundary conditions

$$
Y_{0}\left(\frac{T^{*}}{1-\gamma^{-1}}\right)=1, \quad Y_{0}(1)=0 .
$$

The second step involves the analysis of solutions of the problem (4.6),(4.7) from the perspective of dynamical systems. That is, analysis of trajectories $Y_{0}\left(P_{0}\right)$ on the phase plane $\left(Y_{0}, P_{0}\right)$. Using standard shooting arguments one can show that two trajectories satisfying Eq.(4.6) and one of the boundary conditions (4.7) with different values of $c_{0}$ can not intersect. The latter argument proves the uniqueness of the trajectory. Alternatively, the Theorem 4.1 can be proved by constructing solutions of problem (4.4) on a bounded interval and passing to the limit as the length of the interval goes to infinity [14].

It is important to note, that the result identical to the result of Theorem 4.1 was obtained in [9] for a system which is a modified version of the problem (4.4). This result was the first rigorous result on subsonic detonation.

In applications, it is often important to analyze behavior of traveling wave solutions in the so called high activation energy limit, that is to study behavior of the problem (4.4) with the nonlinear term given by Eq.(2.3) when activation energy $Z$ is large. For large $Z$ the reaction zone shrinks and the transition layer between fresh and burned gas becomes very narrow see Fig. 5. Thanks to the result of the Theorem 4.1, the analysis of the problem (4.4) can be reduced to the analysis of the problem (4.6), (4.7) on the phase plane $\left(Y_{0}, P_{0}\right)$. As it is shown in [23] it is possible to construct sub a super-solutions of this problem on the phase plane which match in the scale of activation energy $Z$. This crucial observation makes possible an asymptotic separation of variables in the equation (4.6) which, in turn, allows the evaluation of the principal part of the propagation velocity $c_{0}$ :

$$
c_{0}(Z)=\frac{1}{\left(1-\gamma^{-1}\right)}+O\left(\frac{\log Z}{\sqrt{Z}}\right) .
$$

The expression (4.8) was first obtained in [19] using formal asymptotic approach. 

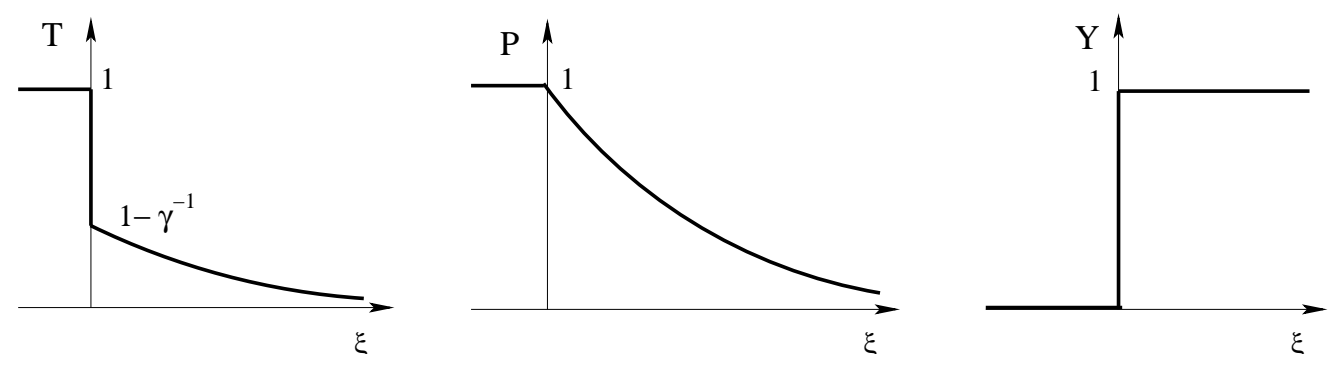

Figure 5: Sketch of the subsonic detonation front in a limit of high activation energy

Moreover, using sub and super-solutions for the problem (4.6) one can estimate the width of the transition layer between fresh and burned gas. In particular, it is possible to show that the thickness of the layer is exponentially small Fig. 6. The latter result strongly suggests
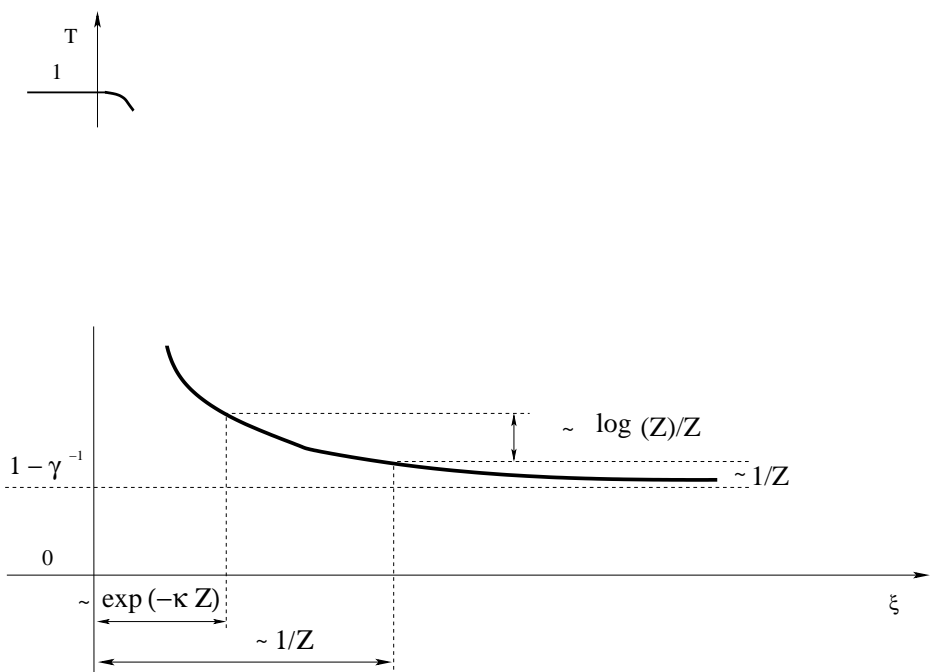

Figure 6: Structure of the transition layer between burned and fresh gases in the limit of large activation energy

that the problem (4.4) converges to the free boundary problem as $Z \rightarrow \infty$. Rigorous proof of this conjecture is still an open problem.

So far our results were concerned with traveling wave solutions for the problem (2.1) under the assumption of the zero thermal diffusivity. In applications, however, the thermal diffusivity $\varepsilon$ is small but finite. Therefore, the zero thermal diffusivity limit needs to be justified. In particular, one can ask the following questions :

- Is there a traveling wave solution for the system (4.2)?

- If so, how many of them?

- What happens with the solutions of the problem (4.2) a $\varepsilon \rightarrow 0$ ? That is, whether there is a convergence of solution as $\varepsilon \rightarrow 0$ ? 
The following three theorems provide a complete answer to these questions.

Theorem 4.2. [24] Let $\varepsilon<1$ and $T^{*}<\lambda_{\varepsilon}$, where $\lambda_{\varepsilon}$ is a positive solution of $\gamma(1-$ $\left.\lambda_{\varepsilon}\right)\left(\gamma+\varepsilon \lambda_{\varepsilon}\right)=1$. The problem (4.2) has a traveling front solution $\left(c_{\varepsilon}, T_{\varepsilon}, P_{\varepsilon}, Y_{\varepsilon}\right)$ with the following properties: $c_{\varepsilon}>0, P_{\varepsilon}, T_{\varepsilon}, Y_{\varepsilon} \in W^{2, \infty}(\mathbb{R}), P_{\varepsilon}, T_{\varepsilon}, Y_{\varepsilon}$ are monotone functions and $0 \leq P_{\varepsilon}, T_{\varepsilon}, Y_{\varepsilon} \leq 1$.

The proof of the theorem follows the general blueprint of [3] with necessary modifications and is based on the construction of a solution on a bounded interval and the subsequent passage to the limit of the whole real line.

Theorem 4.3. [24] Solutions of the problem (4.2) converge uniformly to the unique traveling wave solution of the limiting problem (4.4) as $\varepsilon \rightarrow 0$.

The main ingredient of the proof is to show that the velocity of propagation is bounded from above and from below independently of $\varepsilon$ and that the difference between the solution of the limiting problem (4.4) and solutions of the problem (4.2) is bounded by $\varepsilon$ in $L^{p}(\mathbb{R})$ for $p \geq 1$.

Theorem 4.4. [18] There exist $\varepsilon_{0}>0$ such that if $\varepsilon_{0}>\varepsilon>0$ then the solution of the problem (4.2) is unique.

The proof of this theorem is based on the geometric singular perturbation approach [27].

We have to note that the results of the Theorems 4.2, 4.3 and 4.4 were obtained under the assumption that the Lewis number is equal to unity. However, unlike conventional thermo-diffusive combustion setting $L e \neq 1$ will not change the result.

Theorems 4.2, 4.3 and 4.4 prove that, as conjectured, the thermal diffusivity has indeed a negligible effect on the propagation of detonation fronts. Moreover, since the detonation front is unique at least for $\varepsilon$ small, there is no traveling wave solution corresponding to deflagration wave. As a result, propagation with the velocity close to the velocity of the deflagration front may persist only for a finite time interval. This observation strongly suggests that the transition from deflagration to detonation is unavoidable unless quenching (decay of the solution) occurs.

\section{Propagation and quenching}

The problem of quenching versus propagation has a long history and was first addressed by Kanel [29] in 1964. Kanel considered a problem of extinction and propagation in a context of conventional thermo-diffusive combustion with the Lewis number equal to unity which is described by a single reaction diffusion equation. He considered the problem in one space dimension with initial data given as a characteristic function of the interval.

The problem can be formulated as follows: what initial data will lead to decay of solution, that is solution will approach zero as time goes to infinity, and which give rise to propagation, that is solution approaches a positive constant for a large time on any given compact. Kanel himself gave a partial answer to this question. More specifically, he showed that there exist 
two constants $L_{1}<L_{2}$ such that if the support of initial data is smaller than $L_{1}$ then quenching occurs, and if the support of initial data is larger than $L_{2}$ the propagation is observed. The problem of the sharp bound between extinction and propagation remained open for many years and was recently solved by Zlatos [44] and Matano [35].

The question of distinction between quenching and propagation can also be naturally posed in the context of model (2.1). It turns out that the rough classification between initial data can be obtained. The following two results show that if the initial condition for the temperature is strongly localized then the quenching occurs (Theorem 5.1), whereas initial condition which is larger than the ignition temperature on a sufficiently large support leads to propagation (Theorem 5.2).

The following result provides sufficient conditions for quenching to take place.

Theorem 5.1. [21] Consider problem (2.1) with $\varepsilon<1$ in the entire space $\left(\Omega=\mathbb{R}^{d}\right)$. Assume that the nonlinear term $F(T)$ in addition to be of ignition type satisfies a following bound

$$
F(T) \leq K T
$$

where $K$ is some constant.

$$
\begin{aligned}
& \text { Let } \omega=\left\{\mathbf{x} \in \mathbb{R}^{d}: T_{0}(\mathbf{x})>0\right\} \text { and suppose } \\
& \qquad\left\|T_{0}\right\|_{L^{\infty}} \operatorname{Vol}(\omega) \leq T^{*}\left(\frac{2 \pi d}{K \gamma e}\right)^{d / 2}(\gamma \varepsilon)^{\frac{d}{2}\left(\frac{d}{2}+2\right)}
\end{aligned}
$$

Then,

$$
T(t, \mathbf{x}), P(t, \mathbf{x}) \rightarrow 0 \quad \text { and } \quad Y(t, \mathbf{x}) \rightarrow 1 \quad \text { as } \quad t \rightarrow \infty \text { uniformly in } \quad \mathbf{x}
$$

The proof of the theorem consists of two steps. First we construct the super-solution for the temperature $T$ that obeys a single reaction diffusion equation so that the comparison principle can be used. Employing the approach suggested in [13], we show that at some time $t=t^{*}$ the temperature (super-solution for the temperature) will be below the ignition temperature in entire space. Due to the comparison principle, the temperature then stays below the ignition one for all times $t>t^{*}$. Thus, the super-solution will obey a linear heat equation for $t>t^{*}$. The latter observation leads to the desired result.

Details of the proof of the Theorem 5.1 also provide a transparent physical picture of how quenching occurs. There are two stages of the evolution involved. The first stage is associated with a fast reaction in a localized region of space and an intense transport of heat from the reaction zone. Indeed, initially the pressure is constant and the amount of fuel is abundant while the region of high temperature is extremely localized in space. The reaction raises pressure and temperature in a localized reaction zone and leads to consumption of fuel. At the same time, diffusion prevents high gradient of temperature and eliminates sharp peaks by rapid transport of hot gas away from the reaction zone. Thus after some (relatively short) time the reaction zone is surrounded by a large spot of preheated gas. However, under 
the assumptions of the theorem, the temperature of this preheated gas is still smaller than the ignition temperature. This prevents initiation of the reaction in a larger region. In other words, at the instance of time, estimated from above by $t^{*}$, the temperature drops below the ignition temperature, and the reaction stops in the entire space. Moreover, since $t^{*}$ is finite, just a finite amount of fuel is consumed by this time. At this moment, the second stage of the evolution starts. Hereafter, the dynamics of the system is governed exclusively by diffusion. Diffusion tends to smooth out the pressure and temperature fields that approach zero as time progresses. At the same time, diffusion brings the fuel from infinity in order to make the concentration field uniform and equal to unity at large times.

As an alternative to quenching, one can show that when the initial data for the temperature is large enough and has a large support, one observes a propagation with positive speed in the sense of Aronson and Weinberger [1]. Namely the following theorem holds,

Theorem 5.2. [21] Consider problem (2.1) in the entire space $\left(\Omega=\mathbb{R}^{d}\right)$. Assume that $\varepsilon<1$, Le $\geq\left(1-\lambda_{\varepsilon}\right)^{-1}$, and

$$
T(0, \mathbf{x}) \geq\left\{\begin{array}{cll}
1 & \text { for } & |\mathbf{x}| \leq C \varepsilon^{-1 / 2} \\
\sigma(|\mathbf{x}|) & \text { for } & C \varepsilon^{-1 / 2}<|\mathbf{x}| \leq 1+C \varepsilon^{-1 / 2} \\
0 & \text { for } & |\mathbf{x}|>1+C \varepsilon^{-1 / 2}
\end{array}\right.
$$

where $C$ is some constant independent of $\varepsilon, \lambda_{\varepsilon}$ is a positive solution of $\left(1-\lambda_{\varepsilon}\right)\left(\gamma+\varepsilon \lambda_{\varepsilon}\right)=1$ and $\sigma(z) \in C^{0}$ with $\sigma\left(C \varepsilon^{-1 / 2}\right)=1, \sigma\left(C \varepsilon^{-1 / 2}+1\right)=0$. Assume also that

$$
T^{*}<\min \left(1, \frac{1-\lambda_{\varepsilon}}{\left(\operatorname{Le}\left(1-\lambda_{\varepsilon}\right)\right)^{d / 2}}\left(\frac{1-\varepsilon\left(1-\lambda_{\varepsilon}\right)}{1-\varepsilon\left(1-\lambda_{\varepsilon}\right)^{2}}\right)\right) .
$$

Then, there exist a constant $A>T^{*}$ such that

$$
\lim _{t \rightarrow \infty} \min _{|\mathbf{z}-\mathbf{y}| \leq c t} T(t, \mathbf{z})>A
$$

for any $c \in\left(0, c^{*}\right)$ where $c^{*} \sim \sqrt{\varepsilon}$.

The proof of the theorem is based on the construction of a sub-solution for the temperature which obeys a single reaction diffusion equation and applying arguments of Aronson and Weinberger [1] to the equation describing sub-solution.

The result of the theorem proves that, in case of initial data having relatively large support, quenching is impossible, and propagation with the velocity having at least scaling of deflagration is observed. On the other hand, the result on uniqueness of traveling front and numerical simulations suggest that once propagation has been successfully initiated the reaction will propagate with the characteristic velocity of the order of unity for large times. The proof of this conjecture is an open problem.

Let us note that both quenching and deflagrative propagation are governed by thermal diffusivity $\varepsilon$. Therefore, these effects can not be possibly described in the framework of the zero thermal diffusivity limit, that is by the system (3.5). On the other hand, the absence of thermal diffusivity gives rise to a new regime which can be called lock of propagation. In this 
regime, the solution approaches some nontrivial equilibrium state as $t \rightarrow \infty$. This regime seems to be rather unphysical. Indeed, one can expect that at large times either quenching or propagation is observed. However, in the framework of the zero thermal diffusivity limit this regime makes perfect sense. Formally the system (3.5) can be considered as an intermediate time asymptotic of the system (3.8). That is the system (3.5) approximates the dynamics of the system (3.8) on a time interval of the order $1 / \sqrt{\varepsilon}$. From this perspective, the lock of propagation indicates that fast formation of detonation wave is impossible. Therefore, lock of propagation means that at a time of the order $1 / \sqrt{\varepsilon}$ the bulk burning rate does not exceed $\sqrt{\varepsilon}$. The following result gives a necessary condition for lock of propagation in the dimension one.

Theorem 5.3. [22] Consider system (3.5) with $\Omega=\mathbb{R}$. Assume also that the function $F(T)$ is bounded for all $T$ and $M=\max F(T)$.

Let $T_{0}(x) \geq 0$ and set $\omega=\left\{x \in \mathbb{R}: T_{0}(x)>0\right\}$ and suppose

$$
\operatorname{Vol}(\omega) \leq \frac{T^{*} \sqrt{2 \pi}}{3\left(1-\gamma^{-1}\right) \sqrt{M}}
$$

Then,

$$
\begin{aligned}
& P(t, x) \rightarrow 0 \quad \text { as } t \rightarrow \infty, \quad \text { uniformly in } x \\
& Y(t, x) \rightarrow Y_{\infty}(x) \leq 1 \quad \text { as } t \rightarrow \infty \\
& T(t, x) \rightarrow T_{\infty}(x) \geq T_{0}(x) \quad \text { as } \quad t \rightarrow \infty
\end{aligned}
$$

and

$$
\begin{aligned}
& Y_{\infty}(x)=0, \quad T_{\infty}(x)=\gamma^{-1}+T_{0}(x), \quad \text { if } \quad T_{0}(x)>T^{*}, \\
& Y_{\infty}(x)=1, \quad T_{\infty}(x)=0, \quad \text { if } \quad T_{0}(x)=0 .
\end{aligned}
$$

Moreover,

$$
\lim _{t \rightarrow \infty} Y(t, x) F(T(t, x))=0, \quad \text { for all } \quad x
$$

The proof of the theorem is based on the construction of a super-solution for pressure $P$ which under assumption (5.7) on the initial data approaches zero as $t \rightarrow \infty$.

Numerical simulations show that under certain conditions detonation fronts may become unstable which leads to a rapid oscillations with a large amplitude. This observation gives rise to a natural question whether the bulk burning rate can diverge as $t \rightarrow \infty$. It turns out that in both cases $\varepsilon=0$ and $\varepsilon>0$ this situation is impossible and the bulk burning rate or at least its long time average stays bounded. In order to investigate the long time behavior it is convenient to introduce an average and a long time average of the bulk burning rate. These quantities are defined as follows [31]:

$$
\langle V\rangle_{t}=\frac{1}{t} \int_{0}^{t} V(s) d s
$$




$$
\langle V\rangle_{\infty}=\limsup _{t \rightarrow \infty}\langle V\rangle_{t}
$$

The following two theorems show that under reasonable assumptions on the nonlinearity $F$ and on the initial data $T_{0}(\mathbf{x})$ the long time average of the bulk burning rate $\langle V\rangle_{\infty}$ is bounded independently of $\varepsilon$.

Theorem 5.4. [20] Consider the problem (3.5) with $\Omega=\mathbb{R}$. Assume also that the nonlinear term $F(T)$ is bounded for all $T$ and set $M=\max F(s)$. Suppose that the initial data for the temperate $T_{0}(x)$ has a compact support.

Then

$$
\langle V\rangle_{\infty} \leq 2 \sqrt{\frac{\left(1-\gamma^{-1}\right) M}{T^{*}}}
$$

We have to note that result of the Theorem 5.4 also holds in case where $\Omega$ is a cylinder. Moreover, the result is also applicable to the case where the initial data is not compactly supported but approaches zero sufficiently fast as $x \rightarrow \pm \infty$.

Theorem 5.5. [17] Consider problem (3.8) with $\Omega$ being a cylinder in $\mathbb{R}^{d}$. Assume that the nonlinear term satisfies the bound

$$
F(T) \leq K T
$$

where $K$ is some constant.

Then there exists a constant $\bar{V}$ independent of $\varepsilon$ such that

$$
\langle V\rangle_{\infty} \leq \bar{V}
$$

The proof of the Theorems 5.4 and 5.5 is based on the construction of unbounded supersolutions which, however, decay exponentially at either $x \rightarrow \infty$ or $x \rightarrow-\infty$.

\section{Stabilization of the reaction in a presence of the cold boundary}

In this section, we will discuss the problem of ignition and stabilization of the reaction in porous media in the presence of the cold boundary. The problem in the context of thermo-diffusive combustion was formulated by Zeldovich and Frank-Kamenezky in [15] and first considered by Gelfand and Barenblatt in [16]. The problem is widely known in the mathematical literature as Gelfand's problem. We will use assumptions similar to those in [16] to derive the system of equations which is a natural generalization of the Gelfand problem. So the problem can be called a Gelfand problem for a porous media.

Consider a porous medium filled with the fuel (that is bounded domain $\Omega$ ) embedded in free space. We will assume that on the boundary of the porous matrix the temperature 
and pressure are constant (cold boundary). Depending on the initial conditions on the temperature, there are two possibilities of the development of the reaction process. The first possibility involves an intensive reaction. In this case, the cold boundary does not prevent an active reaction process in the porous media which leads to the rapid rise of the temperature and the pressure up to the point when there is no combustible component left. This type of reaction is called self ignition. The second possible scenario occurs when a reaction process is compensated by the cold boundary. In this case, after some very short time, the nontrivial equilibrium state may be observed. Thus the question is under which conditions the second possibility takes place. In the second case, under certain assumptions on the parameters of the system, we can ignore the fact that the fuel is consumed on some time interval, that is assume that the concentration is constant and equal to the initial one. Taking this into account and rescaling the time and space coordinates in such a way that the size of the domain $\Omega$ occupied by the porous media is fixed, we obtain the following system

$$
\begin{aligned}
& u_{t}=\bar{\varepsilon} \Delta u+\mu \phi(w), \\
& v_{t}=\Delta v+\mu \phi(w), \\
& w(t, \mathbf{x})=(1-h) u(t, \mathbf{x})+h v(t, \mathbf{x}),
\end{aligned}
$$

where $h=\lambda_{\varepsilon} / 1-\bar{\varepsilon}, \mu$ is a scaling parameter and $\lambda_{\varepsilon}$ is a positive solution of $\left(1-\lambda_{\varepsilon}\right)\left(\gamma+\varepsilon \lambda_{\varepsilon}\right)=$ 1. The nonlinear term $\phi$ is identical to the one considered by Gelfand, that is, $\phi(w)$ is positive increasing and convex on $\mathbb{R}$ and super-linear as $w \rightarrow \infty$. A relevant physical example of the nonlinearity is $\phi(w)=e^{w}$. The boundary and initial conditions for the system (6.1) read,

$$
u(0, \mathbf{x})=\frac{1}{1-\lambda_{\varepsilon}} w_{0}(\mathbf{x}), \quad v(0, \mathbf{x})=\frac{\bar{\varepsilon}}{1-\lambda_{\varepsilon}} w_{0}(\mathbf{x})
$$

and

$$
u(t, \mathbf{x})=0, \quad v(t, \mathbf{x})=0 \quad \mathbf{x} \in \partial \Omega .
$$

The question can then be formulated as follows: whether solutions of the system (6.1) with the boundary and initial conditions (6.2) and (6.3) stay bounded for all times or blows up. If solutions stay bounded, one may deduce that the chemical process has indeed stabilized. In contrast, the blow up indicates necessary conditions for the self ignition to take place.

It is obvious that the question of global existence for solutions of the problem (6.1) is related to the existence of stationary solutions. As can be easily verified by direct substitution, if a stationary solution of the problem (6.1) with initial conditions (6.3) exists, then it must satisfy the single elliptic equation

$$
\frac{\bar{\varepsilon}}{1-\lambda_{\varepsilon}} \Delta w_{s}+\mu \phi\left(w_{s}\right)=0 .
$$

The solutions of the problem (6.4) are well understood. In particular, the following results hold [8],[11], [16], [26], [30]. 
There exists $0<\mu^{*}<\infty$ such that for every $0<\mu<\mu^{*}$, equation (6.4) has a minimal, positive classical solution, which is a unique stable solution of (6.4); for $\mu=\mu^{*}$ the problem (6.4) has a weak solution and for $\mu>\mu^{*}$, there is no solutions of the problem (6.4).

These results suggest the following conjecture: If $0<\mu \leq \mu^{*}$ then the problem (6.1) with the initial data $u_{0}=v_{0}=0$ has a global classical solution. Moreover, if $\mu>\mu^{*}$ then any solution of the problem (6.1) with $u_{0}, v_{0} \geq 0$ blows up in finite time.

The conjecture is known to be true for a single parabolic equation even in a much stronger sense [8], [34]. However, to the best of our knowledge, there is no such results for systems.

It is important to note that one may expect that the dynamics of the system (6.1) is close to the dynamics of a single parabolic equation provided by the fact the system (6.1) can be formally rewritten as a gradient flow. Indeed, introducing an energy functional

$$
\mathcal{E}(u, v)=\int_{\Omega} \bar{\varepsilon}(1-h)|\nabla u|^{2}+h|\nabla v|^{2}+\Phi(w), \quad \Phi(w)=-\int_{0}^{w} \phi(s) d s
$$

one can easily verify that the system (6.1) is identical to the following one

$$
(1-h) u_{t}=-\frac{\delta \mathcal{E}}{\delta u}, \quad h v_{t}=-\frac{\delta \mathcal{E}}{\delta v}
$$

As a matter of fact the latter observation allows to obtain a rough classification of the initial data which lead to blow up and of those ensuring bounded solution in the system (6.1). However, the sharp distinction between the two is still an open problem.

Acknowledgments. This work was supported in a part by NSF grant DMS 0554775.

\section{References}

[1] D.G. Aronson, H.F. Weinberger. Multidimensional nonlinear diffusion arising in population genetics. Adv. Math., 1978, Vol. 30, 33-76.

[2] A. Bayliss, B.J. Matkowsky. Two routes to chaos in solid fuel combustion. SIAM J. Appl. Math., 1990, Vol. 50, 437-459.

[3] H. Berestycki, B. Nicolaenco, B. Scheurer. Traveling wave solutions to combustion models and their singular limits. SIAM J. Math. Anal., 1983, Vol. 16, 1207-1242.

[4] I. Brailovsky, V. Goldshtein, I. Shreiber, G. Sivashinsky. On combustion waves driven by diffusion of pressure. Combust. Sci. and Tech., 1997, Vol. 124, 145-165.

[5] I. Brailovsky, G. Sivashinsky. Momentum loss as a mechanism for deflagration to detonation transition. Combust. Theory Modelling, 1998, Vol. 2, 429-447.

[6] I. Brailovsky, G. Sivashinsky. Hydraulic resistance and multiplicity of detonation regimes. Combust. Flame, 2000, Vol. 122, 130-138. 
[7] I. Brailovsky, G.I. Sivashinsky. Effects of momentum and heat losses on the multiplicity of detonation regimes. Combust. Flame, 2002, Vol. 128, 191-196.

[8] H. Brezis, T. Cazenave, Y. Martel, A. Ramiandrisoa. Blow up for $u_{t}-\Delta u=g(u)$ revised. Adv. Differential Equations, 1996, Vol. 1, No. 1, 73-90.

[9] H. Brezis, S. Kamin, G. Sivashinsky. Initiation of subsonic detonation. Asymptotic Analysis, 2000, Vol. 24, 73-90.

[10] V.V. Bychkov, M.A. Liberman. Dynamics and stability of premixed flames. Phys. Reports, 2000, Vol. 325, 115-237.

[11] X. Cabre. Extremal solutions and instantaneous blow-up for elliptic and parabolic problems. Preprint, 2005.

[12] P. Constantin, A. Kiselev, A. Oberman, L. Ryzhik. Bulk burning rate in passive reactive diffusion. Arch. Rational Mech. Anal., 2000, Vol. 154, 53-91.

[13] P. Constantin, A. Kiselev, L. Ryzhik. Quenching of flames by fluid advection. Comm. Pure Appl. Math., 2001, Vol. LIV, 1320-1342.

[14] F. Dkhil. Travelling wave solutions in a model for filtration combustion. Nonlinear Analysis, 2004, Vol. 58, 395-415.

[15] D.A. Frank-Kamenezky. Diffusion and heat transfer in chemical kinetics. Moskow, 1947.

[16] I.M. Gelfand. Some problems in the theory of quasilinear equations. Amer. Math. Soc. Trans., 1963, Vol. 29, 295-381.

[17] A. Ghazaryan, P. Gordon. The KPP type flame fronts in porous media. Preprint, 2007.

[18] A. Ghazaryan, P. Gordon, C.K.R.T. Jones. Traveling waves in porous media combustion: uniqueness of waves for small thermal diffusivity. J. Dynamics Diff. Equations, 2007, Vol.19, 951-966.

[19] I. Goldfarb, G. Kuzmenko, V. Goldshtein. Structure of a pressure driven flame in porous media. Phys. Lett. A, 1999, Vol. 251, 394-403.

[20] P.V. Gordon. An upper bound of the bulk burning rate in porous media combustion. Nonlinearity, 2003, Vol. 16, 2075-2082.

[21] P. Gordon. Quenching and propagation of combustion fronts in porous media. Comm. Math. Sci., Vol. 4, 471-479.

[22] P.V. Gordon, S. Kamin, G.I. Sivashinsky. On initiation of subsonic detonation in porous media combustion. Asymptotic Analysis, 2002, Vol. 29, 309-321. 
[23] P.V. Gordon, L.S. Kagan, G.I. Sivashinsky. Fast subsonic combustion as a free interface problem. Interfaces and Free Boundaries, 2003, Vol. 5, 47-62.

[24] P. Gordon, L. Ryzhik. Traveling fronts in porous media combustion: existence and a singular limit. Proceedings of the Royal Society A, 2006, Vol. 462, 1965-1985.

[25] P.V. Gordon, G.I. Sivashinsky. Pressure diffusivity and low-velocity detonation. Combust. Flame, 2004, Vol. 136, No. 4, 440-444.

[26] M.G. Grandall, P.H. Rabinowitz. Some continuation and variational method for positive solutions of nonlinear elliptic eigenvalue problems. Arch. Rational Mech. Anal., 1975, Vol. 58, 207-218.

[27] C.K.R.T. Jones. Geometric singular perturbation. In Dynamical Systems, Springer Lecture Notes Math. 1609, 1995, 44-120.

[28] Ya. I. Kanel. Some problems involving burning-theory equations. Soviet Math. Dokl., 1961, Vol. 2, 48-51.

[29] Ya. I. Kanel, Stabilization of the solutions of the equations of combustion theory with final initial functions. Mat. Sb., 1964, Vol. 65, 398-413.

[30] H.B. Keller, J. Keener. Positive solutions of convex nonlinear eigenvalue problems. J. Diff. Eq., 1974, Vol. 16, 103-105.

[31] A. Kiselev, L. Ryzhik. An upper bound for the bulk burning rate for systems. Nonlinearity, 2001, Vol. 14, 1297-1310.

[32] G.A. Lyamin, A.V. Pinaev. Fast subsonic combustion of gases in an inert porous medium with smooth rise in the pressure in the wave. Combust. Expl. Shock Waves, 1987, Vol. 23, 399-402.

[33] G.A. Lyamin, V.V. Mitrofanov, A.V. Pinaev, V.A. Subbotin. Propagation of gas explosion in channels with uneven walls and in porous media. In Dynamics of Detonation in Gases and Dispersed Media (A.A. Borisov ed). Kluwer Acad. Pub. Dordrecht, 1991, 51-75.

[34] Y. Martel. Compleate blow up and global behaviour of solutions of $u_{t}-\Delta u=g(u)$., Ann. Inst. Henri Poincare, 1998, Vol. 15, 687-723.

[35] H. Matano. Private communication

[36] B. Matkowsky, G. Sivashinsky. An asymptotic derivation of two models in flame theory associated with constant density approximation. SIAM J. Appl. Math., 1979, Vol. 37, 686-699. 
[37] V.V. Mitrofanov. Non-classical waves of detonation and deflagration. In Mechanics and Scientific-Technological Progress (L.I. Sedov and G.G. Chernyi, eds). Nauka Pub., Moscow, 1987, Vol. 2, 226-242.

[38] G. Sivashinsky. Some developments in premixed combustion modeling. Proc. of the Combustion Inst., 2002, Vol. 29, 1737-1761.

[39] A.A. Vasiliev, V.V. Mitrofanov, M.E. Topchiyan. Detonation waves in gases. Combust. Expl. Shock Waves, 1987, Vol. 23, 605-623.

[40] A.I. Volpert, V.A. Volpert, V.A. Volprt. Traveling wave solutions of parabolic systems. Trans. Math. Monogr., Vol. 140, Amer. Math. Soc., Providence, R.I., 1994.

[41] F. Williams. Combustion Theory. The fundamental theory of chemically reacting flow systems. Perseus Books, Reading, MA, 1985.

[42] J. Xin. Front Propagation in Heterogeneous media. SIAM Rev., 2000, Vol. 42, 161-230.

[43] Y.B. Zeldovich, G.I. Barenblatt, V.B. Librovich, G.M. Makhviladze. Mathematical Theory of Combustion and Explosion. Plenum, New York, 1985.

[44] A. Zlatos. Sharp transition between extinction and propagation of reaction. J. Amer. Math. Soc., 2006, Vol. 19, 251-263. 